\title{
Comportement à la fatigue cyclique de disques en biocéramiques sollicités en compression diamétrale
}

\author{
Samir Hamza ${ }^{1,2,3, a}$, Guy Pluvinage ${ }^{2}$, Zitouni Azari ${ }^{2}$, Joseph Gilgert ${ }^{2,4}$ \\ ET Noureddine Slimane ${ }^{1}$ \\ 1 Laboratoire de Recherche en Biomécanique et Biomatériaux Orthopédique, Institut KASSAB, Ksar Saïd, Tunisie \\ 2 Laboratoire de Fiabilité Mécanique de l'ENIM, Île de Saulcy, 57045 Metz, France \\ 3 Institut National des Sciences Appliquées et de Technologie de Tunis, BP 676, 1080 Tunis Cedex, Tunisie \\ 4 École Nationale d'Ingénieurs de Metz (ENIM), Île de Saulcy, 57045 Metz, France
}

Reçu le 25 janvier 2003, accepté le 2 juillet 2004

\begin{abstract}
Résumé - L'objectif de ce travail est l'étude du comportement à la fatigue cyclique de disques sollicités en compression diamétrale en biocéramiques orthopédiques d'alumine et de zircone $\left(\mathrm{Al}_{2} \mathrm{O}_{3}, \mathrm{ZrO}_{2}\right)$ utilisées pour la conception des prothèses ostéo-articulaires. Nous étudions, grâce aux calculs numériques, l'état de contraintes sur un cotyle d'une prothèse totale de hanche en se basant sur les travaux de Pauwels [Pauwels, Biomécanique de la hanche saine et pathologique, principe, technique et résultats d'une thérapeutique causale, Springer-Verlag, Berlin, Heidelberg, New York, 1977]. La zone la plus sollicitée en contrainte de traction potentiellement à l'origine d'une rupture par fatigue est déterminée. Nous rassemblons des résultats sur la propagation et l'amorçage des fissures de fatigue sous chargement cyclique en compression sur des éprouvettes de simulation de type disque brésilien chargées en mode I et en mode mixte I+II.
\end{abstract}

Mots clés : Fatigue / fissure / amorçage / propagation / compression / biocéramique / prothèse

\begin{abstract}
Behaviour of bioceramic disks submitted to a diametral compressive loading. This research aims to study the propagation of fatigue crack under compression loading in zirconia and alumina bioceramics $\left(\mathrm{Al}_{2} \mathrm{O}_{3}, \mathrm{ZrO}_{2}\right)$ used as femoral heads in total hip replacements (THR). Our aim is to improve lifetime and reliability of bioceramics joint prostheses. In the first part of the paper, we study, using Finite Element Analysis, the stress concentrations around the cup of a total hip prosthesis on the basis of the Pauwels works [Pauwels, Biomécanique de la hanche saine et pathologique, principe, technique et résultats d'une thérapeutique causale, Springer-Verlag, Berlin, Heidelberg, New York, 1977]. The most loaded zone in tension, preferential area of potential crack initiation, has been identified. In the second part are collected results on propagation and initiation of fatigue cracks under compression cyclic loading in brazilian disks loaded in mode I and in mixed mode I+II.
\end{abstract}

Key words: Fatigue / crack / initiation / propagation / compression / bioceramic / prostheses

\section{Introduction}

L'évaluation de la fiabilité des prothèses ostéoarticulaires en biocéramique soumises à des chargements répétés fait appel à des analyses de mécanique de la rupture prenant en compte l'existence de défauts ou de fissures dans les pièces constituant la prothèse (tête et cotyle). La connaissance des cinétiques de propagation de fissure dans ces pièces est nécessaire pour effectuer des analyses et comprendre les causes de rupture par fatigue. La conduite de nos essais de mesure de la vitesse de propagation de fissure tient compte de l'évolution des moyens et méthodes d'essais actuels.

\footnotetext{
a Auteur correspondant : samir.hamza@insat.rnu.tn
}

En arthroplastie totale de hanche les matériaux dits 《 classiques » polyéthylène (Co-Cr-Mo, acier inoxydable 316L...) conservent leur crédit malgré leurs défauts et leurs limites. Ils donnent tout leur intérêt aux recherches actuelles [2-18] qui visent à l'utilisation de matériaux ou couples de matériaux «nouveaux » tels que : céramiquepolyéthylène, céramique-céramique et alliage de titane $\mathrm{TiAl}_{6} \mathrm{~V}_{4}$.

Pour des applications biomédicales, l'évaluation de la durée de vie des biomatériaux et des pièces en service est capitale. Un des risques majeurs de rupture de prothèses en biocéramique est la rupture par fatigue. Les caractéristiques de cette rupture sont les suivantes : amorçage dans une zone de concentration de contrainte et propagation d'une fissure de fatigue. 
Tableau 1. Caractéristiques mécaniques des matériaux étudiés (CARMA, Marseille).

\begin{tabular}{|c|c|c|}
\hline Caractéristiques mécaniques & $\mathrm{Al}_{2} \mathrm{O}_{3}$ & $\mathrm{ZrO}_{2}$ \\
\hline Pureté en \% & 99,9 & 95,6 \\
\hline Résistance à la compression $[\mathrm{MPa}]$ & $\cong 4000$ & $\cong 6000$ \\
\hline Résistance à la flexion [MPa] & $550-600$ & 2200 \\
\hline Module d'Young [GPa] & 380 & 220 \\
\hline Coefficient de Poisson $(\nu)$ & 0,28 & 0,31 \\
\hline Taille moyenne du grain $[\mu \mathrm{m}]$ & 1,3 & 0,35 \\
\hline Masse volumique [g.mm ${ }^{-3}$ ] & 3,9 & 6,1 \\
\hline Ténacité statique en traction, $K_{\mathrm{IC}}[\mathrm{MPa} \sqrt{m}]$ & $3,5-6$ & $6-12$ \\
\hline
\end{tabular}

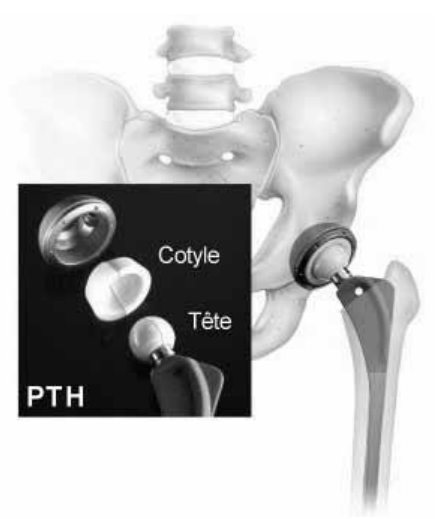

Fig. 1. Prothèse Totale de Hanche (PTH), avec couple céramique-céramique.

Dans cette étude nous prenons le cas de la liaison coxo-fémorale comme exemple significatif (Fig. 1). Le coût élevé des couples en biocéramique n'a pas permis de vérifier sur les pièces réelles le comportement à la fatigue cyclique des prothèses totales de la hanche. Les implants étant sollicités en compression, la propagation des fissures se fait essentiellement par traction induite. Ce mode de sollicitation conduit à une propagation de fissure sensible à trois facteurs : l'amplitude du facteur d'intensité de contraintes $(\Delta K)$, l'amplitude maximale du facteur d'intensité de contraintes $\left(K_{\mathrm{MAX}}\right)$, et la fermeture de fissure par un mécanisme de type « shielding ».

Ce mode de fissuration est également très sensible à l'état microstructural des biocéramiques et conduit notamment à des fissurations intergranulaires. Ceci est d'autant plus grave que la fissuration par fatigue conduit très souvent à une rupture brutale qui provoque un accident. Dans de nombreux cas pratiques, le stade d'amorçage peut être très important (jusqu'à $90 \%$ de la vie d'une pièce cas des céramiques). L'étude de la propagation de fissure visera à établir les lois de propagation dans les biocéramiques et les paramètres de nature intrinsèque, extrinsèque et microstructurale qui l'affectent [19-22].

\section{Matériaux utilisés}

Les biocéramiques se caractérisent par un comportement purement élastique, avec un module d'Young élevé et ce jusqu'à ce que la contrainte à rupture soit atteinte

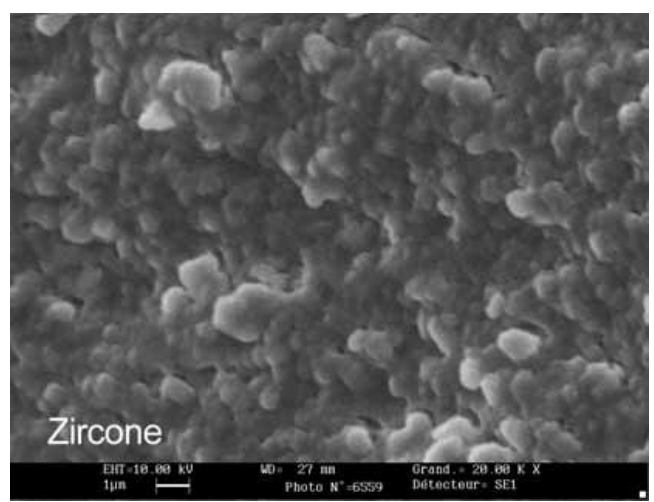

Fig. 2. Image des grains de la zircone utilisée.

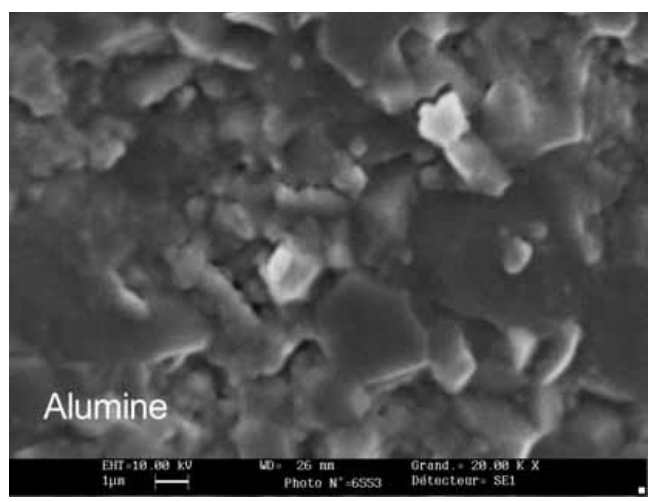

Fig. 3. Image des grains de l'alumine utilisée.

(Tab. 1). Elles ne présentent que de faibles déformations à la rupture. Bien que les biocéramiques présentent une rupture de type « fragile», certaines d'entre elles peuvent résister à des contraintes de compression très élevées, c'est le cas de la zircone, même malgré une absence totale d'accommodation de la plasticité sous l'effet des concentrations de contraintes [23].

L'alumine, même avec une microstructure fine et une grande pureté chimique (99,9\%), présente une résistance à la rupture modeste pour une céramique. Elle est donc sensible aux défauts et présente donc une faible résistance aux concentrations de contraintes. La granulométrie de la biocéramique est caractérisée par un diamètre moyen de $0,35 \mu \mathrm{m}$ pour la zircone et $1,3 \mu \mathrm{m}$ pour l'alumine (Figs. 2 et 3$)$.

La qualité d'une biocéramique dépend de sa pureté, de sa densité, de sa porosité, de la taille de ses grains, 


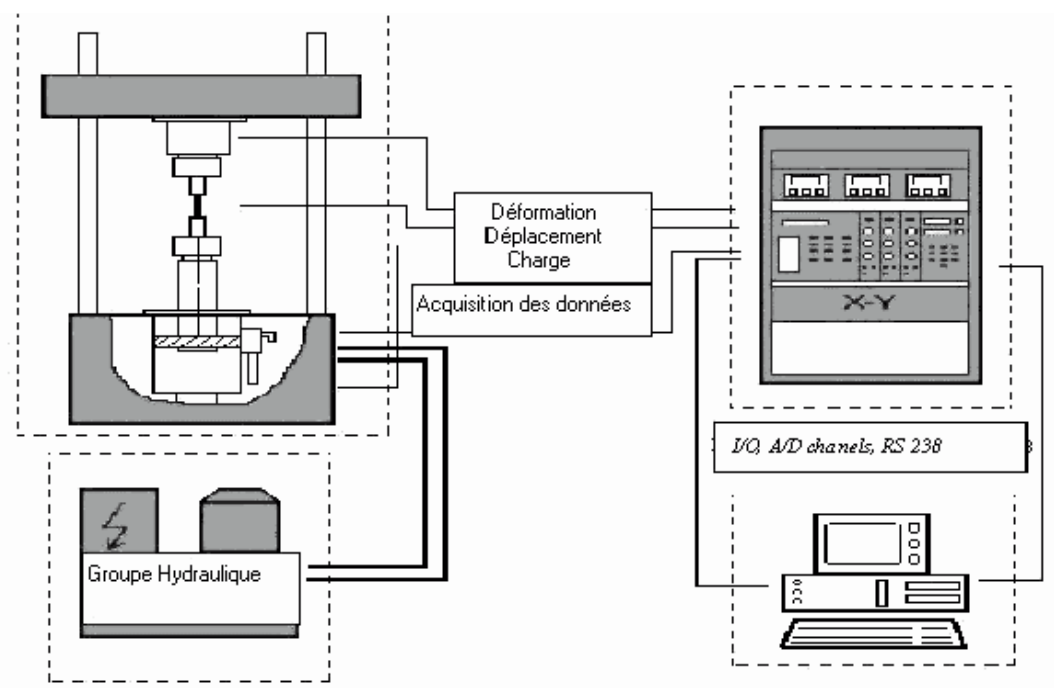

Fig. 4. Schéma de principe de fonctionnement de la machine INSTRON utilisée pour la conduite des essais de fatigue en compression.

Tableau 2. Valeur de l'amplitude de la charge $\Delta P$ appliquée sur les éprouvettes.

\begin{tabular}{lcc}
\hline & $\begin{array}{c}\text { Disque brésilien } \\
\text { chargé en mode I } \\
\left(\beta=0^{\circ}\right)\end{array}$ & $\begin{array}{c}\text { Disque brésilien } \\
\text { chargé en mode I+II } \\
\left(\beta=34^{\circ}\right)\end{array}$ \\
\hline Éprouvette en alumine & $10 \mathrm{kN}$ & $7 \mathrm{kN}$ \\
Éprouvette en zircone & $16 \mathrm{kN}$ & $13 \mathrm{kN}$ \\
Nombre d'éprouvettes & 4 en alumine & 4 en alumine \\
testées & et 4 en zircone & et 4 en zircone \\
\hline
\end{tabular}

de sa structure cristalline, de sa résistance en flexion et à la fatigue et de ses tolérances.

Les procédés de fabrication des éprouvettes par frittage sont extrêmement précis. Selon la qualité de cette fabrication les caractéristiques mécaniques des éprouvettes et les résultats observés sont aussi variables.

\section{Technique expérimentale}

Les implants étant sollicités en compression, la propagation des fissures se fait essentiellement par traction induite. Pour effectuer ces essais, nous disposons d'une machine de fatigue de traction-compression servohydraulique type INSTRON de capacité $\pm 100 \mathrm{kN}$ qui présente un excellent alignement de l'éprouvette sur l'axe de travail afin d'éviter les risques de cisaillement. Cette machine est équipée d'un capteur de déplacement dont la sensibilité est de l'ordre du micromètre. Le résultat dépend énormément de la qualité de l'alignement et de l'état de surface des éprouvettes.

L'architecture globale du système de contrôle et d'asservissement de la machine INSTRON est donnée par le schéma de la figure 4, qui décrit la partie active du système et les différentes conditions pour le contrôle de la charge, du déplacement et de déformation.
Tous les essais ont été conduits en compression cyclique sinusoïdale à température ambiante, à la fréquence de $30 \mathrm{~Hz}$ et pour un rapport de chargement $R=$ $\sigma_{\min } / \sigma_{\max }=10$ (Tab. 2).

Nous avons eu recours à des essais de compression diamétrale, dits aussi essais brésiliens, pour évaluer la contrainte de traction qui gouverne l'amorçage et la propagation des fissures de fatigue $[24,25]$. Cet essai, qui convient plus particulièrement aux céramiques, consiste à comprimer uniaxialement un disque de $40 \mathrm{~mm}$ suivant une direction diamétrale. Les essais sur disques brésiliens ont été conduits en mode $\mathrm{I}\left(\beta=0^{\circ}\right)$, et en mode $\mathrm{I}+\mathrm{II}$ $\left(\beta=34^{\circ}\right)$ (Fig. 5).

Le mode I de rupture est supposé être le mode le plus dangereux. Pour simplifier le problème sur le plan expérimental, il est admis que les chargements unidirectionnels appliqués à des éprouvettes de type disque brésilien peuvent reproduire totalement ou partiellement des conditions réelles de chargement.

L'avancement de la fissure est suivi sur les deux faces du disque brésilien à l'aide d'un système de visée optique bidimensionnel (lunettes) de grossissement égal à 50, monté sur une table à déplacements micrométriques à l'avant et d'une mini-caméra à l'arrière de l'éprouvette (Fig. 6).

Les essais réalisés ont nécessité un soin minutieux pour le montage des éprouvettes. Un résultat satisfaisant a 


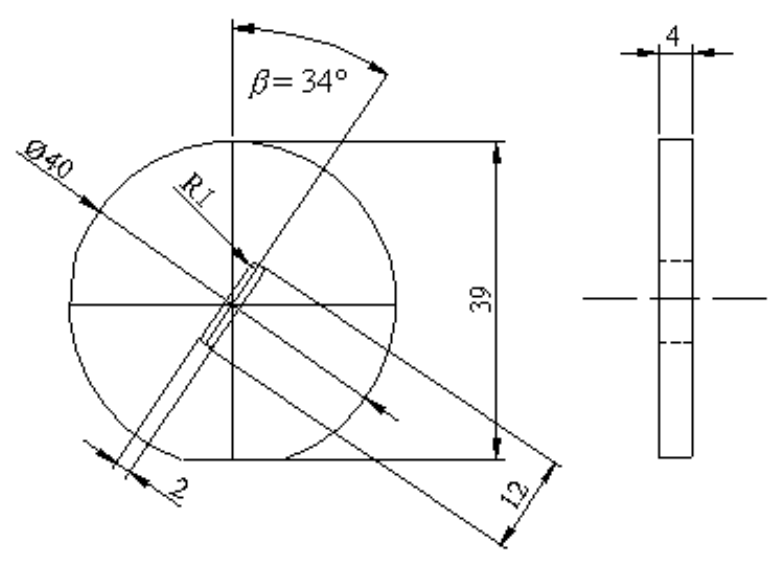

Fig. 5. Géométrie des éprouvettes de type disques brésilien utilisées. Rayon du fond d'entaille $R_{1}=1 \mathrm{~mm}$.

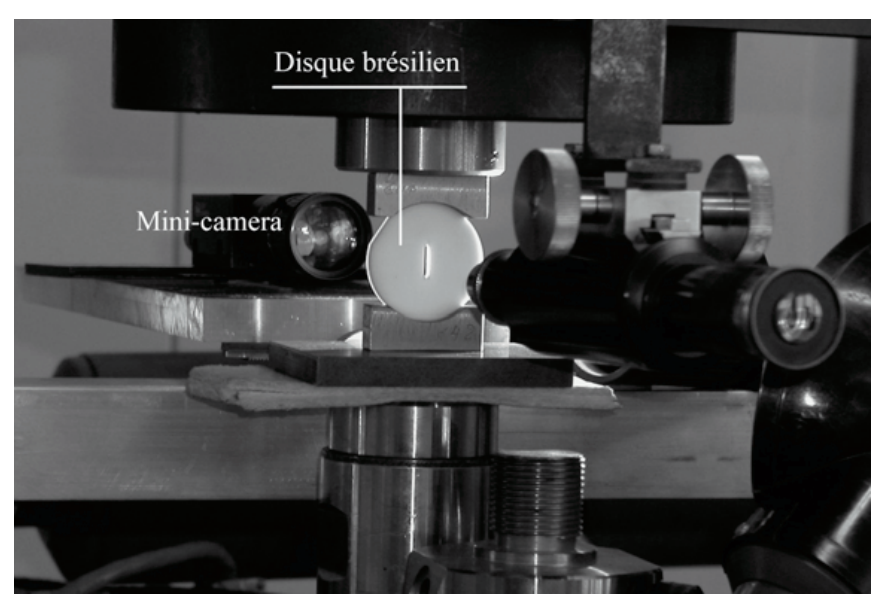

Fig. 6. Montage de chargement utilisé pour la conduite des essais de fatigue en compression sur disque brésilien et montage du suivi de la vitesse de fissuration : lunette optique à l'avant de l'éprouvette et mini-camera CCD à l'arrière de l'éprouvette.

été obtenu après plusieurs tentatives infructueuses. Au cours de cette étude, nous avons été confrontés à des problèmes majeurs : fragilité des matériaux, coût élevé des éprouvettes et des essais, mode de chargement en compression difficile à conduire et sensibilité à la rupture brutale des céramiques. Un soin tout particulier a porté sur le choix du montage pour assurer une parfaite symétrie de chargement et de fissuration sur les éprouvettes.

\section{Résultats de l'analyse par éléments finis}

La modélisation numérique a été menée entièrement sur le logiciel Castem, afin de déterminer les distributions des contraintes et le calcul des facteurs d'intensité de contrainte. Le but de cette modélisation est d'optimiser le dimensionnement des éléments d'une prothèse totale de hanche en biocéramique d'alumine et de zircone afin d'éviter ou de réduire les champs des contraintes élevées.

Nous avons utilisé des éléments triangulaires isoparamétriques à six nœuds. Le maillage en pointe de fissure est particulièrement affiné pour la modélisation en approche locale, la concentration des contraintes y étant très élevée. Le même type de maillage a été conservé pour toutes les éprouvettes en alumine et en zircone en mode I et en mode I+II afin d'éliminer toute influence de changement du maillage sur les résultats obtenus. L'exploitation des résultats se trouve également facilitée puisqu'on peut obtenir les contraintes, les déformations et les déplacements en chaque nœud.

Le matériau est considéré avoir un comportement isotrope élastique et les contraintes se déduisent aisément des déformations (et vice versa) à l'aide de la relation de Hooke généralisée :

$$
\frac{1+\nu}{E} \sigma_{i j}=\varepsilon_{i j}+\frac{\nu}{1-2 \nu}(\text { Trace } \tilde{\varepsilon}) \delta_{i j}
$$

où $\delta_{i j}$ représente le symbole de Kronecker et la trace $\tilde{\varepsilon}=$ $\varepsilon_{11}+\varepsilon_{22}+\varepsilon_{33}$.

Les conditions aux limites sont traitées par la méthode des multiplicateurs de Lagrange. Elles s'écrivent sous la forme suivante :

$$
\overline{\bar{C}} \bar{u}=\bar{q}
$$

Elles sont prises en compte dans le système d'équations linéaires d'équilibre en résolvant le système suivant :

$$
\left\{\begin{array}{c}
\overline{\bar{K}} \bar{u}+\overline{\bar{C}} \bar{\lambda}=\bar{F} \\
\overline{\bar{C}} \bar{u}=\bar{q}
\end{array}\right.
$$

avec :

$\overline{\bar{C}}$ : tenseur des modules élastiques;

$\overline{\bar{K}}$ : matrice de rigidité;

$\{u\}$ : vecteur des déplacements généralisés;

$\{F\}$ : vecteur des forces généralisées;

$\{q\}$ : vecteur colonne des forces imposées (forces nodales).

- La rigidité $\overline{\bar{C}}$ est à adjoindre à la rigidité $\overline{\bar{K}}$ du système libre grâce à l'opérateur BLOQUER.

- Le vecteur $\bar{q}$ est à adjoindre au vecteur des forces nodales $\bar{F}$ grâce à l'opérateur DEP (MPOSE).

La définition du chargement consiste à créer un champ par points correspondant au vecteur du second membre de l'équation :

$$
\overline{\bar{K}} \bar{u}=\bar{F}
$$

Une fois la préparation du modèle de calcul réalisée, on constitue le système :

$$
\overline{\bar{M}} \overline{\ddot{u}}+\overline{\bar{C}} \overline{\dot{u}}+\overline{\bar{K}} \bar{u}=\bar{F}
$$

Par la suite le calcul est effectué par l'intermédiaire du logiciel Castem.

Notre étude est basée sur les travaux effectués par Pauwels [1] sur le chargement du bassin : lorsqu'on représente la sollicitation mécanique de la tête fémorale sur le cotyle, on part d'un état d'équilibre statique du corps. Le cotyle est essentiellement sollicité en compression. L'intensité de la force de compression agissant sur 


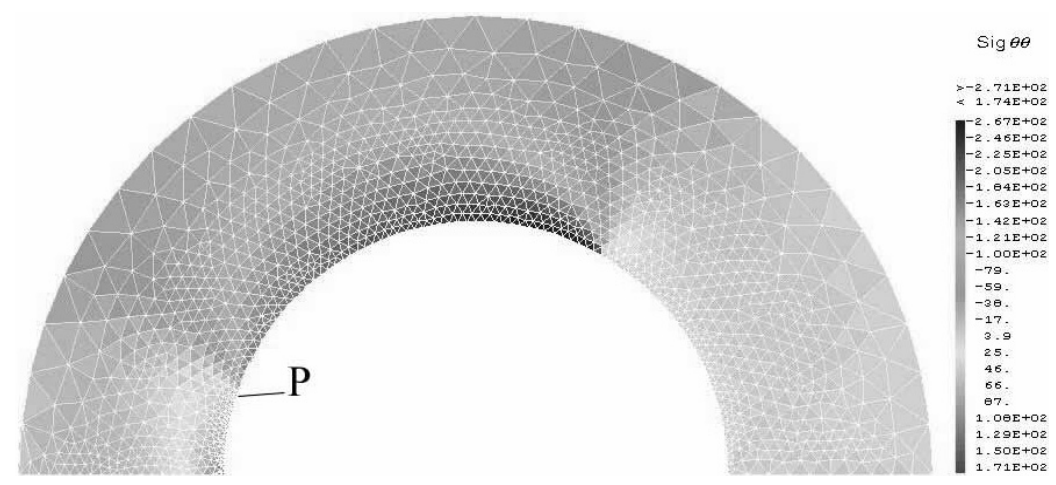

Fig. 7. Distribution des contraintes $\sigma_{\theta \theta}[\mathrm{MPa}]$ sur un cotyle d'une prothèse totale de hanche.

le cotyle et répartie sur un secteur angulaire durant la période d'appui unilatéral, est environ 4 fois la masse du corps, soit $280 \mathrm{~kg}$ pour une personne pesant $70 \mathrm{~kg}$ et qui se tient sur une seule jambe. La valeur de $2,8 \mathrm{kN}$ est choisie pour effectuer nos calculs par éléments finis sur l'élément cotyle d'une prothèse totale de hanche.

Les conditions aux limites : encastrement des segments de la base, et du grand cercle (Fig. 7), application de l'effort conformément aux travaux de Pauwels [1]. Nous avons procédé au calcul des contraintes locales $\sigma_{r r}, \tau_{r \theta}$ et $\sigma_{\theta \theta}$ pour localiser les points les plus sollicités de l'élément cotyle au niveau du contact avec la tête de la PTH.

Les plages foncées représentent les contraintes en compression et les plages claires représentent les contraintes en traction. La valeur de la contrainte diminue suivant la profondeur dans le cotyle. Les plages claires sont les plus sollicitées. À cet endroit, les contraintes sont de traction et favorables à l'amorçage d'une fissure de fatigue. Nous concluons que la rupture des cotyles se produit en traction indirecte préférentiellement à partir du point $\mathrm{P}$ (Fig. 7).

L'analyse des contraintes au niveau du contact tête et cotyle n'a pas été l'objectif principal de la modélisation. Elle s'est attachée à reproduire le plus fidèlement possible ce qui se passe dans le cotyle d'une prothèse totale de hanche.

Nous avons déterminé l'évolution des contraintes $\sigma_{r r}$ et $\sigma_{\theta \theta}$ en fonction de la distance $r$ de la pointe de l'entaille mécanique pour un chargement en mode $\mathrm{I}+\mathrm{II}\left(\beta=34^{\circ}\right)$ et pour un chargement en mode $\mathrm{I}\left(\beta=0^{\circ}\right)$ (Fig. 8). Nous remarquons qu'au niveau du point $\mathrm{P}$, la contrainte de traction est la plus élevée pour les deux biocéramiques étudiées. L'amorçage de la fissure par fatigue prend donc naissance en ce point.

Nous avons déterminé par la suite les valeurs des facteurs d'intensités de contraintes $K_{\mathrm{I}}$ et $K_{\mathrm{II}}$ lorsque le disque est sollicité en mode mixte I+II $\left(\beta=34^{\circ}\right)$, et la valeur de $K_{\text {I }}$ lorsque le disque est sollicité en mode I $\left(\beta=0^{\circ}\right)$ par calcul aux éléments finis et par la méthode analytique d'Atkinson [26,27]. Les résultats obtenus pour un chargement en mode I et en mode mixte I+II, sont regroupés dans le tableau 3 .

L'affinement du maillage au niveau du point considéré, induit une variation des valeurs de $K_{\mathrm{I}}$ et $K_{\text {II }}$ obtenus par le code de calcul Castem. Il est loin d'être

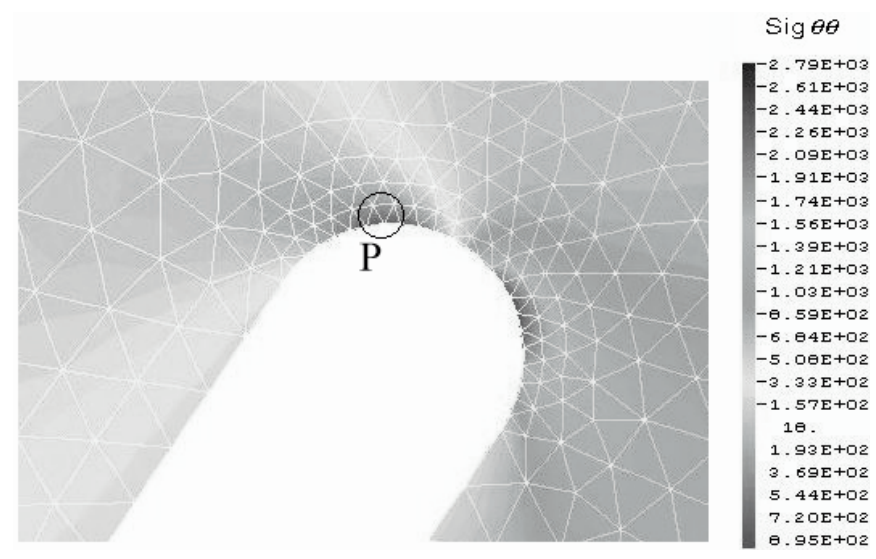

Fig. 8. Exemple de distribution des contraintes $\sigma_{\theta \theta}[\mathrm{MPa}]$ sur un disque brésilien en zircone sous un chargement de $16 \mathrm{kN}$, chargé en mode I+II $\left(\beta=34^{\circ}\right)$. Agrandissement au niveau $\mathrm{du}$ fond de l'entaille. Les plages foncées : zone en traction; les plages claires : zone en compression.

certain que le calcul analytique approché donne une valeur des $K_{\mathrm{I}}$ et de $K_{\mathrm{II}}$ plus exacte que celle fournie par les éléments finis : les méthodes numériques ont permis d'affiner considérablement les valeurs des $K$ dans des cas de chargement trop complexes pour permettre un calcul analytique rigoureux.

Les calculs aux éléments finis sur des éprouvettes du type disque brésilien permettent de choisir la nature du chargement (analogue à celui du cotyle) et de bien connaître la distribution des contraintes au niveau de la fissure (Tab. 4).

Le choix préférentiel qui s'est porté sur des éprouvettes du type disque brésilien est consécutif aux difficultés rencontrées lors des essais sur les éprouvettes du type CT [28]. En outre, elles conduisent à des essais en mode mixte s'approchant au mieux des conditions réelles du comportement en service des prothèses de hanche.

L'angle $\beta=34^{\circ}$ est l'angle le plus approprié pour simuler la bifurcation car il conduit à un angle de bifurcation sous sollicitation en mode I+II $\left(\beta=34^{\circ}\right)$ de $\psi=36,1^{\circ}$. Cet angle $\psi$ correspond au même angle de bifurcation sur le cotyle $\psi=36^{\circ}$.

Nous remarquons que le mode $\mathrm{I}\left(\beta=0^{\circ}\right)$ est le mode qui s'approche le mieux des conditions réelles de 
Tableau 3. Valeurs de $K_{\mathrm{I}}$ et de $K_{\mathrm{II}}$ obtenues pour les éprouvettes type disque brésilien en alumine et en zircone par la méthode d'Atkinson et par calcul Castem.

\begin{tabular}{|c|c|c|c|c|c|c|c|}
\hline \multirow[b]{3}{*}{$\Delta P[\mathrm{kN}]$} & \multirow[b]{3}{*}{ Méthode utilisée } & \multicolumn{3}{|c|}{ Éprouvette en alumine } & \multicolumn{3}{|c|}{ Éprouvette en zircone } \\
\hline & & \multirow{2}{*}{$\begin{array}{c}\text { Mode I } \\
\beta=0^{\circ} \\
K_{\mathrm{I}}\end{array}$} & \multicolumn{2}{|c|}{$\begin{array}{c}\text { Mode I+II } \\
\beta=34^{\circ}\end{array}$} & \multirow{2}{*}{$\begin{array}{c}\text { Mode I } \\
\beta=0^{\circ} \\
K_{\mathrm{I}}\end{array}$} & \multicolumn{2}{|c|}{$\begin{array}{c}\text { Mode I+II } \\
\beta=34^{\circ}\end{array}$} \\
\hline & & & $K_{\mathrm{I}}$ & $K_{\mathrm{II}}$ & & $K_{\mathrm{I}}$ & $K_{\mathrm{II}}$ \\
\hline \multirow[t]{2}{*}{10} & Castem & 5,60 & & & & & \\
\hline & Atkinson & 6,89 & & & & & \\
\hline \multirow[t]{2}{*}{7} & Castem & & $-3,14$ & 4,74 & & & \\
\hline & Atkinson & & $-2,37$ & 6,91 & & & \\
\hline \multirow[t]{2}{*}{16} & Castem & & & & 9,27 & & \\
\hline & Atkinson & & & & 11,03 & & \\
\hline \multirow[t]{2}{*}{13} & Castem & & & & & $-5,92$ & 8,69 \\
\hline & Atkinson & & & & & $-3,86$ & 12,84 \\
\hline
\end{tabular}

Pour $\beta=0^{\circ}$ la valeur de $K_{\mathrm{II}}$ est égale à zéro $\left(K_{\mathrm{II}}=0\right)$, mode I de chargement. $K_{\mathrm{I}}$ et $K_{\mathrm{II}}$ sont en $[\mathrm{MPa} \sqrt{m}]$.

Tableau 4. Tableau récapitulatif des contraintes principales et angle de bifurcation.

\begin{tabular}{lccc}
\hline & Élément du cotyle & $\begin{array}{c}\text { Disque brésilien } \\
\text { chargé en mode I } \\
\left(\beta=0^{\circ}\right)\end{array}$ & $\begin{array}{c}\text { Disque brésilien } \\
\text { chargé en mode I+II } \\
\left(\beta=34^{\circ}\right)\end{array}$ \\
\hline $\begin{array}{l}\text { Angle de bifurcation } \\
\text { de la fissure }\left(\psi^{\circ}\right)\end{array}$ & $36^{\circ}$ & $0^{\circ}$ & $36,1^{\circ}$ \\
$\sigma_{11}[\mathrm{MPa}]$ & 61,52 & 556 & 304,11 \\
$\sigma_{22}[\mathrm{MPa}]$ & $-105,09$ & -1112 & $-759,42$ \\
$\begin{array}{l}\text { Rapports des contraintes } \\
\text { principales } \sigma_{11} / \sigma_{22}\end{array}$ & $-0,58$ & $-0,50$ & $-0,40$ \\
\hline
\end{tabular}

chargement sur le cotyle $\left(\sigma_{11} / \sigma_{22}=-0,58\right)$. En définitive nous pensons que cette condition est plus impérative car nous nous sommes préoccupés particulièrement de l'amorçage.

\section{Résultats et discussion}

\section{1 Évolution de la longueur de fissure (a) en fonction du nombre de cycles $\left(N_{\mathrm{p}}\right)$ sur des disques brésiliens chargés en mode I $\left(\beta=0^{\circ}\right)$}

Ce type d'essai est difficile à mettre en ouvre pour des biocéramiques. Par ailleurs, les courbes obtenues sont sensibles à la disposition des éprouvettes et à l'application de la charge.

Avant d'aborder l'étude de fissuration de fatigue en mode mixte I+II, nous avons tout d'abord caractérisé la propagation en mode I. Le but de cette étude est de conduire des essais de fatigue qui simulent le plus fidèlement possible les conditions de chargement au niveau des cotyles des prothèses.

Lors des essais en mode mixte I+II $\left(\beta=34^{\circ}\right)$, nous n'avons pas observé le phénomène de propagation. La rupture est brutale après un nombre de cycles très élevé pour l'amorçage. Par contre, dans le cas de chargement

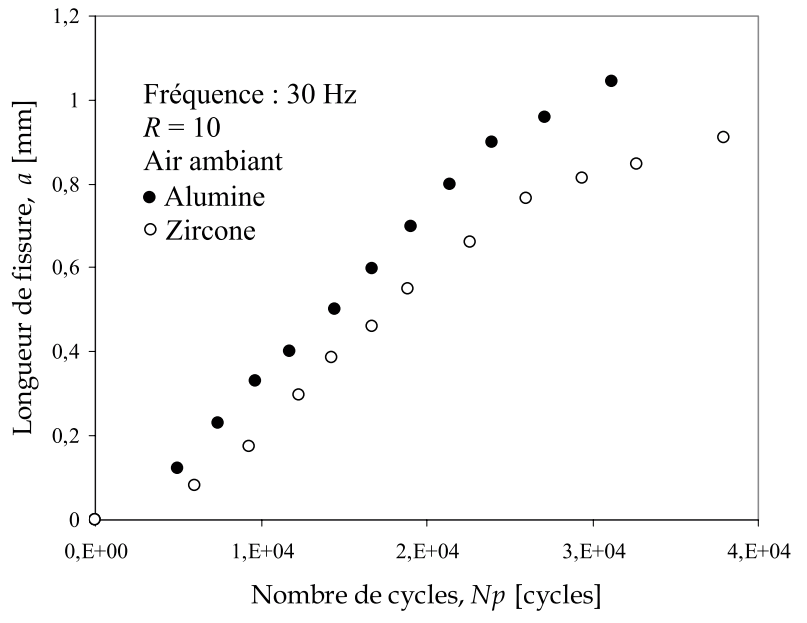

Fig. 9. Évolution du diagramme $\left(a, N_{\mathrm{p}}\right)$ sur les disques brésiliens chargés en compression diamétrale.

en mode I $\left(\beta=0^{\circ}\right)$, l'amorçage et la propagation sont contrôlés.

Les évolutions de la longueur des fissures $a$ en fonction du nombre de cycles $N_{\mathrm{p}}$ sont présentées par les diagrammes de la figure 9. La propagation des fissures en mode I a lieu sans bifurcation. 


\section{2 Évolution de la propagation des fissures de fatigue en fonction de $\Delta K_{1}$ et $K_{1 \text { Max }}$ pour les disques brésiliens chargés en mode I $\left(\beta=0^{\circ}\right)$}

Pour les disques brésiliens chargés en mode I pur, le facteur d'intensité de contrainte $K_{\mathrm{I}}$ est exprimé par un facteur $N_{\text {I }}$ sans dimension [27-29], tel que :

$$
N_{\mathrm{I}}=\frac{K_{\mathrm{I}}}{K_{0}}
$$

où :

$$
\begin{aligned}
K_{0} & =\sigma_{\mathrm{p}} \sqrt{\pi a} \\
\sigma_{\mathrm{p}} & =\frac{P}{B R \pi}
\end{aligned}
$$

$a=$ demi-longueur de l'entaille;

$B=$ épaisseur du disque ;

$P=$ intensité de la charge;

$R=$ rayon du disque;

$K_{\mathrm{I}}=$ facteur d'intensité de contrainte en mode I pur ;

$\sigma_{\mathrm{p}}=$ contrainte appliquée au centre (mais cette contrainte est pratiquement constante sur tout le diamètre ce qui est une propriété remarquable du disque brésilien).

$$
N_{\mathrm{I}}=\left(1+\frac{3}{2}\left(\frac{a}{R}\right)^{2}+\frac{3}{4}\left(\frac{a}{R}\right)^{4}+\frac{3}{64}\left(\frac{a}{R}\right)^{6}\right)
$$

d'où l'expression de $K_{\mathrm{I}}$ :

$$
K_{\mathrm{I}}=\frac{P}{B R \pi} \sqrt{\pi a}\left(1+\frac{3}{2}\left(\frac{a}{R}\right)^{2}+\frac{3}{4}\left(\frac{a}{R}\right)^{4}+\frac{3}{64}\left(\frac{a}{R}\right)^{6}\right)
$$

Une fois l'ensemble des couples $\left(\mathrm{d} a / \mathrm{d} N, \Delta K_{\mathrm{I}}\right)$ calculés, ils sont reportés sur un diagramme en coordonnées bi-logarithmiques (abscisses : valeurs de $\Delta K_{\mathrm{I}}$ ou $K_{\text {IMax }}$, ordonnées : valeurs de $\mathrm{d} a / \mathrm{d} N)$, figures 10 et 11 , afin de vérifier si les biocéramiques utilisées suivent la loi de fissuration de Paris [28] :

$$
\frac{\mathrm{d} a}{\mathrm{~d} N}=C\left(\Delta K_{\mathrm{I}}\right)^{m}
$$

avec $\Delta K_{\mathrm{I}}=\left(K_{\text {IMax }}-K_{\text {Imin }}\right), m$ et $C$ sont des paramètres qui caractérisent la biocéramique et les conditions d'essai.

Pour la prise en compte du rapport de chargement $R^{\prime}$ par le biais du facteur $K_{\text {IMax }}$, nous avons tracé l'évolution de la vitesse de propagation des fissures de fatigue en fonction de $K_{\text {IMax }}$. Le modèle est de la forme :

$$
\frac{\mathrm{d} a}{\mathrm{~d} N}=C^{\prime}\left(\Delta K_{\mathrm{I}}\right)^{P}\left(K_{\text {IMax }}\right)^{n}
$$

$C^{\prime}, p$ et $n$ sont des facteurs caractéristiques de la biocéramique.

Or

$$
K_{\mathrm{IMax}}=\frac{\Delta K_{\mathrm{I}}}{1-R^{\prime}}
$$

$R^{\prime}=\frac{K_{\min }^{\text {eff }}}{K_{\mathrm{Max}}^{\text {eff }}}:$ rapport de chargement local.

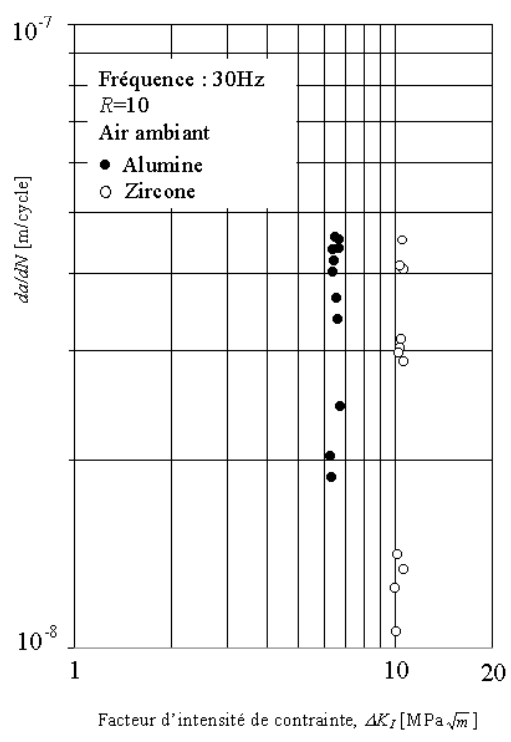

Fig. 10. Diagramme $\left(\mathrm{d} a / \mathrm{d} N, \Delta K_{\mathrm{I}}\right)$ obtenu sous chargement en mode I sur les disques brésiliens pour l'alumine et la zircone.

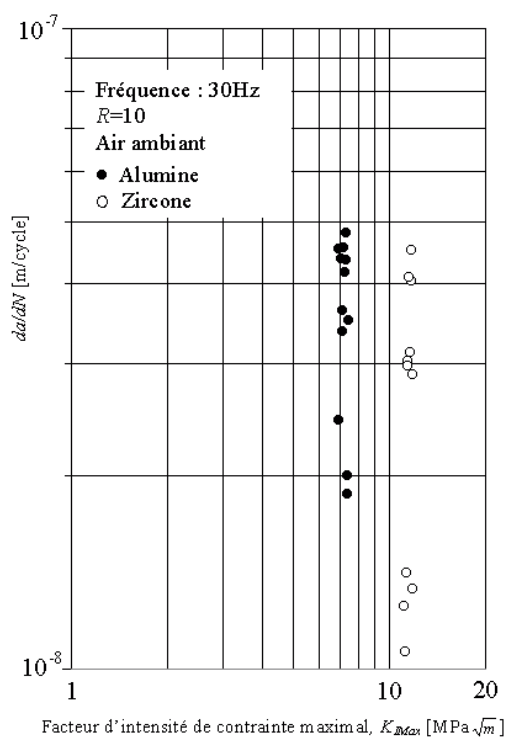

Fig. 11. Diagramme (d $\left.a / \mathrm{d} N, K_{\text {IMax }}\right)$ obtenu sous chargement en mode I sur les disques brésiliens pour l'alumine et la zircone.

À partir de l'équation (12), nous avons l'expression de $\mathrm{d} a / \mathrm{d} N$ en fonction de $\Delta K_{\mathrm{I}}$ :

$$
\begin{aligned}
& \frac{\mathrm{d} a}{\mathrm{~d} N}=\frac{C}{\left(1-R^{\prime}\right)^{n}}\left(\Delta K_{\mathrm{I}}\right)^{(n+p)} \\
& C^{\prime}=C\left(1-R^{\prime}\right)^{n}
\end{aligned}
$$

et

$$
(n+p)=m
$$

Les coefficients $n, p$ et $C^{\prime}$ sont déterminés à partir du diagramme $\mathrm{d} a / \mathrm{d} N$ en fonction du facteur d'intensité de contraintes $K_{\text {IMax }}$ à l'aide de l'équation suivante :

$$
\frac{\mathrm{d} a}{\mathrm{~d} N}=C^{\prime}\left(1-R^{\prime}\right)^{p}\left(K_{\mathrm{IMax}}\right)^{n+p}
$$


Tableau 5. Facteurs $m, C, C^{\prime}, n$ et $p$ et comparaison avec les résultats obtenus par Ritchie dans le cas d'un chargement en mode I.

\begin{tabular}{ccc}
\hline Coeff. & \multicolumn{2}{c}{ Nos résultats } \\
\cline { 2 - 3 } & $\mathrm{Al}_{2} \mathrm{O}_{3}$ & $\mathrm{ZrO}_{2}$ \\
\hline$C$ & $8,3 \times 10^{-19}$ & $5,6 \times 10^{-30}$ \\
$C^{\prime}$ & $0,71 \times 10^{-19}$ & $0,55 \times 10^{-30}$ \\
$n$ & 23,3 & 21,9 \\
$p$ & 6,7 & 5,1 \\
$m$ & 30 & 27 \\
\hline \multicolumn{3}{c}{ Résultats obtenus par Ritchie et al. } \\
\hline$C$ & $5,2 \times 10^{-18}$ & $3,31 \times 10^{-22}$ \\
$C^{\prime}$ & $5 \times 10^{-16}$ & - \\
$n$ & $17-31,6-21.8$ & - \\
$p$ & $1,6-9,8$ & - \\
$m$ & $27, \ldots 33$ & $21, \ldots 42$ \\
\hline
\end{tabular}

Les figures 10 et 11 montrent l'allure de deux courbes obtenues en mode I pour $\beta=0^{\circ}$. Les facteurs d'intensité de contrainte $K_{\mathrm{I}}$ et $K_{\mathrm{IMax}}$ sont obtenus à partir du modèle d'Atkinson [26].

Nous avons caractérisé la propagation des fissures de fatigue en mode I sur l'alumine et la zircone. Nous avons vérifié, pour les deux biomatériaux, que le rapport de charge n'a effectivement aucune influence sur l'évolution de la vitesse moyenne du front de fissure en fonction de $K_{\mathrm{I}}$.

La figure 10 montre que l'intervalle de variation du facteur d'intensité de contrainte en mode $\mathrm{I}\left(\beta=0^{\circ}\right)$ varie entre 6 et $7 \mathrm{MPa} \sqrt{m}$ pour l'alumine et entre 10 et $11 \mathrm{MPa} \sqrt{m}$ pour la zircone pour un intervalle de variation de $\mathrm{d} a / \mathrm{d} N$ entre 1 et $5 \times 10^{-8} \mathrm{~m} /$ cycle. Nous remarquons également sur cette figure que, pour une même valeur de $\mathrm{d} a / \mathrm{d} N$, le facteur d'intensité de contrainte pour la zircone est plus élevé que celui de l'alumine.

À partir des résultats présentés dans les figures 10 et 11 , il convient donc d'estimer les paramètres $C, m$, $C^{\prime}, n$ et $p$ et de contrôler la régression pour tous les diagrammes $\left(\mathrm{d} a / \mathrm{d} N, \Delta K_{\mathrm{I}}\right.$ et $\left.K_{\mathrm{IMax}}\right)$ développés dans cette étude. Le seuil de propagation de fissure est la valeur asymptotique de $\Delta K_{\mathrm{I}}$ et $K_{\text {IMax }}$ pour laquelle $\mathrm{d} a / \mathrm{d} N$ tend vers zéro. Le tableau 5 présente les valeurs de $m, n, p, C$ et $C^{\prime}$ et leur comparaison avec les travaux de Ritchie [30-35].

La différence entre nos résultats et ceux obtenus dans les travaux de Ritchie [30-35] est due à la nature de la céramique étudiée et surtout à la taille des grains. Les valeurs de $n$ sont élevées et $n>p$, ce qui indique une propagation par bonds discrets.

Nous avons constaté, à partir des essais préliminaires, que les fissures aux deux extrémités se propagent de façon symétrique de part et d'autre du disque (Figs. 12-15). Par conséquent, nous nous sommes contentés de n'effectuer les mesures des quantités $\Delta a_{x}$ et $\Delta a_{y}$ que sur un seul côté de chaque front de fissure.

Les figures 12 et 13 montrent que, sur les disques brésiliens testés sous chargement en mode $\mathrm{I}\left(\beta=0^{\circ}\right)$, la fissure ne bifurque pas car la contrainte $\sigma_{\theta \theta}$, ici de

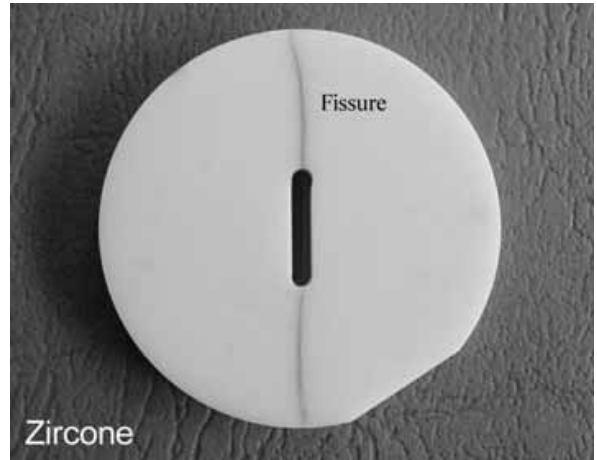

Fig. 12. Disque brésilien en zircone après essai en mode I $\left(\beta=0^{\circ} ; \psi=0^{\circ}\right)$.

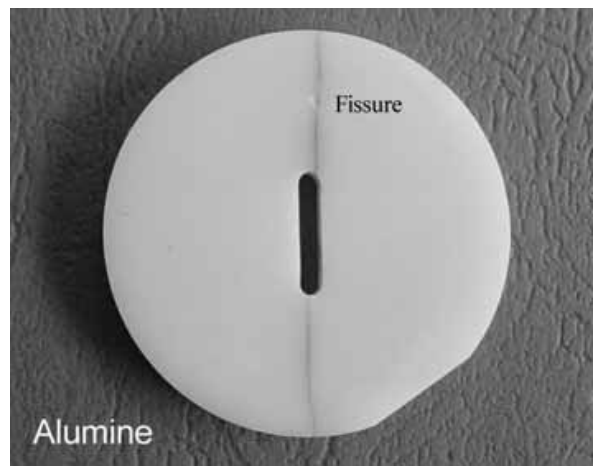

Fig. 13. Disque brésilien en alumine après essai en mode I $\left(\beta=0^{\circ} ; \psi=0^{\circ}\right)$.

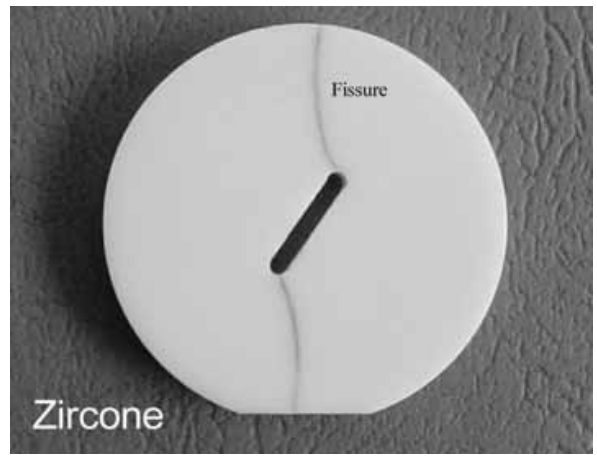

Fig. 14. Disque brésilien en zircone après essai en mode I +II $\left(\beta=34^{\circ} ; \psi=36^{\circ}\right)$.

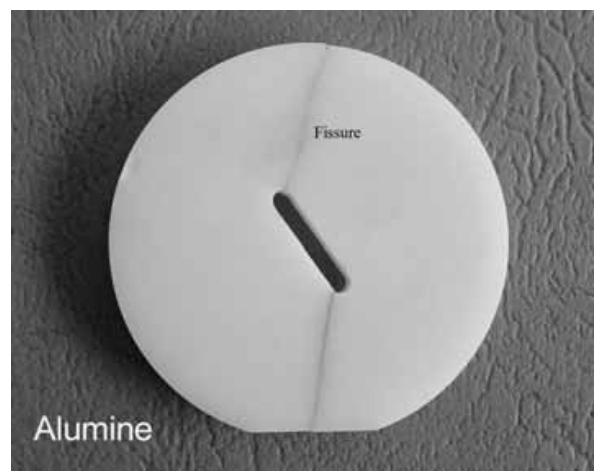

Fig. 15. Disque brésilien en alumine après essai en mode I $+\mathrm{II}\left(\beta=34^{\circ} ; \psi=36^{\circ}\right)$. 
traction, est perpendiculaire à la direction suivie par la fissure. Nous sommes dans le cas où la valeur de la contrainte de traction est maximale.

\section{3 Étude de l'amorçage et de la propagation des fissures de fatigue en compression pour les disques brésiliens chargés en mode I+II $\left(\beta=34^{\circ}\right)$}

Les essais sur les disques brésiliens chargés en compression en mode mixte $\mathrm{I}+\mathrm{II}\left(\beta=34^{\circ}\right)$ ont été conduits dans les mêmes conditions que les essais en mode I ( $\beta=$ $\left.0^{\circ}\right)$. Lors de ces essais, nous n'avons pas pu enregistrer la vitesse de fissuration compte tenu du caractère brutal de la rupture. Les éprouvettes testées, figures 14 et 15, montrent une bifurcation de fissure avec angle de bifurcation $\psi=36^{\circ}$ par rapport au repère lié au fond de la fissure. L'amorçage a eu lieu après $4,9 \times 10^{4}$ cycles sous une amplitude de charge $\Delta P=13 \mathrm{kN}$ pour la zircone, et $3,1 \times 10^{4}$ cycles pour l'alumine sous une amplitude de charge $\Delta P=7 \mathrm{kN}$. La rupture fragile, observée lors de ces essais sur l'alumine et la zircone chargées en mode mixte I+II, implique deux stades :

- initiation d'une fissure ou d'un défaut (amorçage);

- propagation à partir du défaut préexistant le plus sévère. Dans ce stade, la contrainte élevée conduit à une valeur de $K_{\mathrm{I}}$ voisine de $K_{\mathrm{IC}}$ et on se rapproche du régime de rupture catastrophique où la vitesse de fissuration est très grande.

La contrainte normale est négative. La propagation de la fissure s'effectue avec une bifurcation telle que le facteur d'intensité de contrainte diminue (Figs. 14 et 15). Le champ de contrainte est considéré comme biaxial compressif $\sigma_{r r}$ et $\sigma_{\theta \theta}$. Les fissures ne s'amorcent qu'en mode I, avec bifurcation dans la direction de contrainte de traction maximale à leur extrémité.

La différence d'évolution de la loi de Paris entre les deux biocéramiques s'explique par les valeurs des paramètres qui agissent sur la fissuration et qui sont de deux types :

- les paramètres intrinsèques qui dépendent du type de la biocéramique (alumine ou zircone), à savoir le module d'Young (380 GPa pour l'alumine et de $210 \mathrm{GPa}$ pour la zircone), la résistance en compression de la zircone $(\cong 6000 \mathrm{MPa})$ qui est supérieure à celle de l'alumine $(\cong 4000 \mathrm{MPa})$, les propriétés microstructurales (grosseur des grains, porosité ...);

- les paramètres extrinsèques qui dépendent des conditions de l'essai, notamment le rayon en fond d'entaille qui peut légèrement varier.

\section{Conclusions}

Dans l'état actuel de la technique de fabrication par frittage d'une Prothèse Totale de Hanche (PTH), avec couple céramique-céramique, le problème majeur n'est plus le frottement qui est faible $(\mu=0,06$ pour un couple alumine-alumine et $\mu=0,1$ pour un couple aluminepolyéthylène) mais la longévité de la fixation du cotyle qui se trouve mécaniquement dans la situation d'une pièce rigide logée dans une cavité déformable et élastique.

À partir des calculs des sollicitations locales au point le plus chargé du cotyle, on a cherché à reproduire sur des éprouvettes simples et bon marché le système de sollicitations locales au point critique du cotyle. Le choix s'est alors porté sur des éprouvettes de type disques brésiliens chargés en mode I $\left(\beta=0^{\circ}\right)$ et en mode I+II $\left(\beta=34^{\circ}\right)$. Ces éprouvettes conduisent à un mode mixte de fissuration s'approchant au mieux des conditions réelles du comportement en service des prothèses de hanche.

Les essais ont permis de comparer deux types de biocéramiques : l'alumine et la zircone qui sont de très bons biomatériaux pour la fabrication des prothèses à frottement alumine-alumine et alumine-zircone employées en orthopédie. L'avenir de ces produits en prothèses ostéoarticulaires (hanche, genou, épaule, cheville...) semble être prometteur si une meilleure connaissance des critères de résistance à la fatigue de ces biocéramiques permet de garantir une longévité compatible avec les exigences du patient et l'absence d'intervention chirurgicale fréquente.

Nos essais ont montré que la zircone présente une meilleure tenue à la fatigue en compression et une meilleure résistance à la rupture que l'alumine.

L'inconvénient majeur de l'utilisation de la zircone comme élément de friction pour les prothèses de hanche est, d'après les orthopédistes $[4,5,14,17]$, son rôle dans la germination des phosphates de calcium à partir des fluides biologiques sursaturés. Ceci peut provoquer un cancer après un certain temps d'implantation, mais cette hypothèse reste à prouver.

L'utilisation de biomatériaux avec de plus hautes caractéristiques est une des solutions envisagées. Dans le cas des prothèses en orthopédie, les nanocéramiques semblent être la solution pour réaliser des couples à frottement très faible et par voie de conséquence réduire l'amplitude de sollicitation en fatigue par compression.

Remerciements. Le présent travail a été effectué dans le cadre d'une action intégrée (CMCU-00F0808) entre la Faculté de Médecine de Tunis (LRBBIO) et l'Université de Metz (LFM). Les auteurs remercient ces institutions et le Comité Mixte Franco-Tunisien pour leur soutien financier et l'ensemble des collaborateurs qui ont participé à l'accomplissement de cette recherche.

\section{Références}

[1] F. Pauwels, Biomécanique de la hanche saine et pathologique, principe, technique et résultats d'une thérapeutique causale, Springer-Verlag, Berlin Heidelberg, New York, 1977

[2] S. Lerouge, O. Huk-Papanastasiou, L'H. Yahia, J. Witvoet, L. Sedel, Ceramic-Ceramic and MetalPolyethylene Total Hip Replacements: Comparison of pseudomembranes after loosening, J. Bone and Joint Surgery 79(1) (1997) 135-139 
[3] F. Langlais, Prothèses articulaires : facteurs biologiques et mécaniques de tolérance, Cahier d'enseignement de la SOFCOT, conférences d'enseignement, $2^{\mathrm{e}}$ série, 1983, pp. $15-42$

[4] S. Lerouge, O. Huk-Papanastasiou, L'H. Yahia, L. Sedel, Characterization of In Vivo Wear Debris from CeramicCeramic Total Hip Arthroplasties, Biomed. Mat. Res. 32 (1996) 627-633

[5] H. Oonishi, S. Wakitani, N. Murata, M. Saito, K. Imoto, S. Kim, M. Matsuura, Clinical experience with ceramics in total hip remplacement, Clinical Orthopaedics and Related Research 379 (2000) 77-84

[6] F. Prudhommeaux, M. Hamadouche, J. Nevelos, C. Doyle, A. Meunier, L. Sedel, Wear of alumina-alumina total hip arthroplasties at a mean 11-year followup, Clinical Orthopaedics and Related Research 379 (2000) 113-122

[7] P. Boutin, D. Blanquaert, Les nouveaux matériaux utilisés dans les prothèses totales de hanche, Extrait de cahiers d'enseignement de la SOFCOT 10 (1979) 27-44

[8] S. Affatato, M. Goldoni, M. Testoni, A. Toni, Mixed oxides prosthetic ceramic ball heads, Part 3: effect of $\mathrm{ZrO}_{2}$ fraction on the wear of ceramic on ceramic hip joint prostheses, A long-term in vitro wear study, Biomaterials 22 (2001) 717-723

[9] A. Fabre, Simulation de marche pour tester les prothèses de hanche à l'usure : modélisation et réalisation, Mécanique Industrielle et Matériaux 47(3) septembre 1994

[10] P. Boutin, Arthroplastie totale de la hanche par prothèse en alumine frittée, étude expérimentale et premières applications cliniques, Revue de chirurgie orthopédique et réparatrice de l'appareil moteur (Paris) 58(3) (1972) 229-246

[11] B. Calès, Wear behaviour of ceramic pairs compared on different testing configurations, D.E. Marlowe, J.E. Parr, M.B. Mayor (ed.), Modularity of orthopedic implants, Philadelphia : ASTM, 1997, pp. 69-82

[12] L. Sedel, L'alumine en chirurgie orthopédique, Extrait de cahiers d'enseignement de la SOFCOT, $\mathrm{n}^{\circ} 25$, biomatériaux en chirurgie orthopédique, expansion scientifique française, février 1993, pp. 61-69

[13] P. Boutin, P. Chritel, J.M. Dorlot, A. Meunier, D. Blanquaert, S. Herman, L. Sedel, J. Witvoet, The use of dense alumina-alumina ceramic combination in total hip remplacement, John Wiley \& Sons, Inc., J. Biomedical Mat. Res. 22 (1988) 1203-1232

[14] A. Marti, Inert bioceramics $\left(\mathrm{Al}_{2} \mathrm{O}_{3}-\mathrm{ZrO}_{2}\right)$ for medical application, Injury, Int. J. Care Injured 31 (2000) S-D 33-36

[15] G.J.P. Fleming, R.M. Shelton, P.M. Marquis, The influence of clinically induced variability on the bi-axial fracture strength of aluminous core porcelain discs, J. dentistry 27 (1999) 587-594

[16] P. Boutin, Arthroplastie totale de la hanche par prothèse en alumine frittée, étude expérimentale et premières applications cliniques, Revue de chirurgie orthopédique et réparatrice de l'Appareil moteur (Paris) 58(3) (1972) 229-246

[17] S. Affatato, M. Goldoni, M. Testoni, A. Toni, Mixed oxides prothetic ceramic ball heads, Part 3: effect of the $\mathrm{ZrO}_{2}$ fraction on the wear of ceramic on ceramic hip joint protheses, A long-term in vitro wear study, Biomaterials 22 (2001) 717-723
[18] L. Sedel, Evolution of alumina-alumina implants, Clinical Orthopaedics and Related Research, 379 Octobre 2000, pp. $48-54$

[19] S. Hamza, K. Jendoubi, G. Pluvinage, J. Gilgert, N. Slimane, Comportement en fatigue des biocéramiques $\left(\mathrm{Al}_{2} \mathrm{O}_{3}-\mathrm{ZrO}_{2}\right)$ utilisées en orthopédie, $2^{\mathrm{e}}$ Symposium International des Biomatériaux avancés (SIBA'2000), 28 juin $-1^{\text {er }}$ juillet 2000 , Montréal, p. 101

[20] Y. Murakami, Stress intensity factors handbook, Pergamon Press, vol. 1, pp. 991-993

[21] C.J. Gilbert, R.O. Ritchie, Mechanisms of cyclic fatiguecrack propagation in fine-grained alumina ceramic: The role of crack closure, Fatigue Fract. Eng. Mat. Struct. 20 (1997) 1453-1466

[22] S.H. Teoh, Fatigue of biomaterials: a review, International J. Fatigue 22 (2000) 825-837

[23] R.O. Ritchie, Mechanisms of fatigue-crack propagation in ductile and brittle solids, Int. J. Fracture 00 (1998) 1-29

[24] J.P. Henry, J. Pacquet, Présenté par P. Germain, Mécanique de la rupture appliqué aux dépouillements des essais brésiliens sur disques et anneaux, C.R. Acad. Sc. Paris Série B 284(23) (1977) 511-514

[25] Y. Niwa, S. Kobayashi, T. Fukui, Effects of couple stresses distributions in a disc specimen subjected to diametral compression, Mem. Fac. Eng. Kyoto Univ. 33(3) (1971) 118-127

[26] C. Atkinson, Combined mode fracture via the cracked Brazilian disc test, Int. J. Fract. 18(4) (1982) 279-291

[27] M. Louah, Fissuration en fatigue en mode I+II à l'aide du disque brésilien, Thèse de docteur es sciences physiques, Université de Metz 1986

[28] P.C. Paris, The fracture mechanics approach to fatigue, Fatigue, an interdisciplinary approach, Syracuse, University Press, Syracuse, 1964

[29] S. Hamza, Étude du comportement en fatigue en compression des biocéramiques $\left(\mathrm{Al}_{2} \mathrm{O}_{3}, \mathrm{ZrO}_{2}\right)$ utilisées dans la conception des prothèses ostéo-articulaires, Thèse de Doctorat de l'Université de Metz, décembre 2002

[30] R.H. Dauskarat, D.B. Marshall, R.O. Ritchie, Cyclic fatigue- crack propagation in magnesia-partiallystabilized Zirconia Ceramics, J. Am. Ceram Soc. 73(4) (1990) 893-903

[31] R.H. Dauskardt, W. Yu, R.O. Ritchie, Fatigue crack propagation in transformation-toughened Zirconia ceramic, J. Am. Ceram Soc. 70(10) (1987) 248-252

[32] C.J. Gilbert, R.N. Petrany, R.O. Ritchie, Cyclic fatigue in monolithic alumina: mechanisms for crack advance promoted by frictional wear of grain bridges, J. Materials Science 30 (1995) 643-654

[33] A.A. Steffen, R.H. Dauskarat, R.O. Ritchie, Cyclic fatigue life and crack-Growth behavior of microstructurally small cracks in magnesia-partially-stabilized Zirconia Ceramics, J. Am. Ceram Soc. 76(6) (1991) $1259-1268$

[34] C.J. Gilbert, R.H. Dauskardt, R.O. Ritchie, Microstructural Mechanisms of cyclic fatigue-crack propagation in grain-bridging ceramics, Ceramics International 23 (1997) 413-418

[35] R.H. Dauskarat, D.B. Marshall, R.O. Ritchie, Cyclic fatigue- crack propagation in magnesia-partiallystabilized Zirconia Ceramics, J. Am. Ceram Soc. 73(4) (1990) 893-903 